\title{
The Importance of Spatial Distribution of Stemness and Proliferation State in Determining Tumor Radioresponse
}

\author{
H. Enderling ${ }^{1}$, D. Park, L. Hlatky and P. Hahnfeldt \\ Center of Cancer Systems Biology, Caritas St. Elizabeth's Medical Center \\ Tufts University School of Medicine, Boston, 02135, USA
}

\begin{abstract}
Tumor growth and progression is a complex phenomenon dependent on the interaction of multiple intrinsic and extrinsic factors. Necessary for tumor development is a small subpopulation of potent cells, so-called cancer stem cells, that can undergo an unlimited number of cell divisions and which are proposed to divide symmetrically with a small probability to produce more cancer stem cells. We show that the majority of cells in a tumor must indeed be non-stem cancer cells with limited life span and limited replicative potential. Tumor development is dependent as well on the proliferative potential and death of these cells, and on the migratory ability of all cancer cells. With increasing number of cells in the tumor, competition for space limits tumor progression, and in agreement with in vitro observation, the majority of cancer cells become quiescent, with proliferation primarily occurring on the outer rim where space is available. We present an agent-based model of early tumor development that captures the spatial heterogeneity of stemness and proliferation status. We apply the model to simulations of radiotherapy to predict treatment outcomes for tumors with different stem cell pool sizes and different quiescence radiosensitivities. We show by first presuming homogeneous radiosensitivity throughout the tumor, and then considering the greater resistance of quiescent cells, that stem cell pool size and stem cell repopulation during treatment determine treatment success. The results for tumor cure probabilities comprise upper bounds, as there is evidence that cancer stem cells are also more radioresistant than other tumor cells. Beyond just demonstrating the influence of mass effects of stem to non-stem cell ratios and proliferating to quiescent cell ratios, we show that the spatiotemporal evolution of the developing heterogeneous population plays a pivotal role in determining radioresponse and treatment optimization.
\end{abstract}

Key words: cancer, stem cell, radiotherapy, quiescence, proliferation, repopulation AMS subject classification: 92B99

\footnotetext{
${ }^{1}$ Corresponding author. E-mail: heiko.enderling@tufts.edu
} 


\section{Introduction}

Cancer is the abnormal growth of cells that escape tissue control and proliferate abnormally to form a tumor that invades the host tissue. Cancer cells are generally described as a population of cells that have acquired a series of mutations in there genome to express the so-called six hallmarks of cancer: evading apoptosis, limitless replicative potential, self-sufficiency in growth signals, insensitivity to anti-growth signals, sustained angiogenesis and tissue invasion and metastasis [1]. According to a prevailing paradigm for cancer risk estimation, once a cancer cell is created, symptomatic cancer disease will inevitably result [2]. Over the last decade or so, however, we have learned that frank cancers may exist as dormant nodules [3] despite the presence of a subpopulation of tumor stem cells. The first evidence of such cancer initiating cell surfaced for leukemia, tumors of the blood $[4,5]$. Recently, tumorigenic cells have also been associated with solid tumors e.g. of the breast, brain, prostate and colon $[6,7,8,9,10,11,12,13]$. The cancer stem cell hypothesis posits that a small subpopulation of immortal cancer stem cells exist in the tumor with the ability to proliferate indefinitely, and, with a certain probability, divide symmetrically to give rise to more such cancer stem cells. By this theory, the mechanism by which the mortal cancer cells comprising the rest of the tumor are replenished is through asymmetric stem cell division, during which the stem cell maintains its property and the daughter cell is fated to mature [14]. The clinical significance of the stem cell hypothesis is that optimum tumor control is not necessarily achieved by killing the majority of the cancer cells, but by eradicating the small subpopulation of cancer stem cells $[15,16]$. With increasing tumor mass cells compete for limited resources such as space and nutrients. Tumor growth can be inhibited by its own mass [17], lack of oxygen [18, 19] or the microenvironment $[20,21]$. As the tumor grows proliferating cells tend to bias to the tumor periphery and the center becomes more quiescent. Nutrient limitations can give rise to this proliferation gradient, although we and others have shown that cell-density-dependent growth inhibition is also a factor $[22,23]$. Accordingly, renewed growth requires neovascularization, a facilitative microenvironment and, as had been proposed earlier by Norton and colleagues [24, 25], seeding of cells to peripheral areas of low density. Heterogeneity in cellular proliferation status is very important for treatment planning. Radiosensitivity varies considerably throughout the cell cycle, and quiescent cells, i.e. cells in $G_{0}$ phase, are less sensitive than proliferating cells [26, 27]. Cells quiescent at the time of radiation can become proliferative again and repopulate the tumor even beyond pre-treatment cell numbers [28]. Different mathematical models of radiation response have previously considered a homogeneously radiosensitive tumor population [29, 30], as well as tumor populations composed of cells with varying radiotherapeutic $[31,32]$ and chemotherapeutic resistance $[33,34,35]$. Here we present a model that focuses on the understated role of stem cell pool size and stem cell repopulation during the course of treatment. We show that the spatio-temporal evolution of the developing heterogeneous population [36] plays a pivotal role in determining radioresponse and treatment optimization. 


\section{Model}

We use an agent-based model (see [37] for a review of similar model approaches) to simulate tumor development from single cancer stem cells. We let the tumors grow to a certain size, and then simulate different radiotherapy protocols to elucidate the roles of cancer stem cells and spatiotemporal population dynamics in tumor response.

\subsection{Tumor growth model}

Cells are considered as individual entities with a cell cycle, maturation age of one day, migration potential $\mu=0.00635 \mathrm{~mm} \mathrm{~h}^{-1}$ ( $\approx 1$ cell width / 90 minutes) [38] and a limited replication capacity $\rho=\left[0, \rho_{\max }\right]$. A replication capacity of $\rho_{\max }$ means that one cell can divide $\rho_{\max }$ times, producing potentially $2^{\rho_{\max }}$ cells, before reaching senescence and undergoing cell death. In the case of a cancer stem cell, $\rho_{\max }=\infty$. Time is discretized with the smallest unit in our model, i.e. $\Delta t=90$ mins, and increment the age of each cell. Based upon available space cells can migrate, or (if the maturation age has also been reached) divide, reducing $\rho$ by one. Additionally, at each cell division cancer stem cells can with probability $p_{s}$ divide symmetrically to produce another cancer stem cell with identical features, or with probability $1-p_{s}$ divide asymmetrically to produce a cancer stem cell and a non-stem cancer cell with limited proliferation capacity $\rho_{\max }$. Non-stem cancer cells are additionally subject to spontaneous cell death with probability $\alpha /$ day. If the local environment is saturated with cells, i.e. no adjacent lattice points are vacant, we assume cells are unable to migrate or divide to proliferate and place a daughter cell nearby. A cell unable to migrate and proliferate becomes quiescent.

Algorithmically, cells are chosen at random to be updated dependent on their internal state and the local environment. Subject to available space the cell will migrate into a random empty neighboring site or maintain its current position. If the maturation age is reached proliferation is attempted. Without proliferation capacity, i.e. $\rho=0$, the cell dies and is deleted from the simulation. If $\rho>0$ the cell can divide if a neighboring lattice point is available. If more than one of the eight adjacent grid points in two dimensions are free, a lattice site is chosen at random where the daughter cell will be placed. The daughter cell will inherit the parents reduced proliferation capacity or $\rho_{\max }$ if the parent is a stem cell. If the parent is a stem cell, both daughters will become stem cells with probability of symmetric division $p_{s}$. Otherwise, one daughter will become a nonstem cell. A flowchart of the simulation process is shown in figure 1. We solve the model in a twodimensional domain of $3,500 \mu \mathrm{m} \times 3,500 \mu \mathrm{m}$ divided in $350 \times 350$ lattice points of $10 \mu \mathrm{m}^{2}$, each of which can host at the most one cell at any time. The simulations of tumor growth are initialized with a single cancer stem cell in the center of the domain. Once the tumors have reached a specific size, i.e. 100, 000 cells, the simulation is halted and the tumor morphology and age structure saved to simulate and compare different radiotherapy protocols on that tumor. 


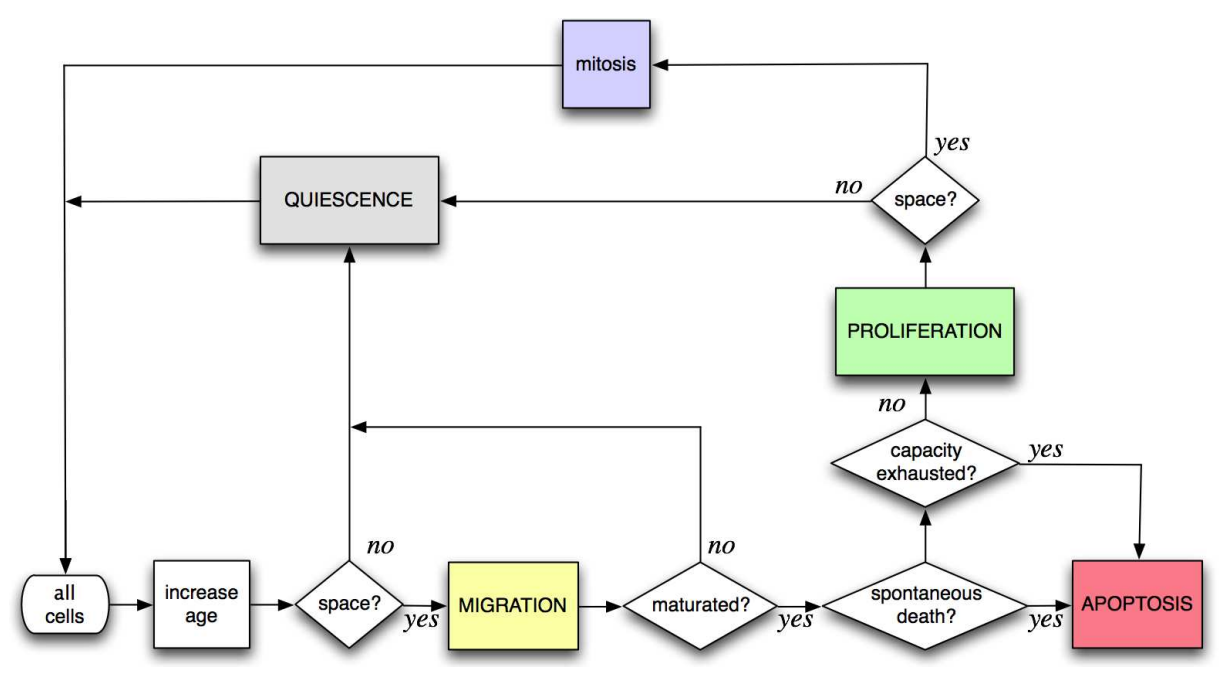

Figure 1: Simulation flowchart and cell life cycle scheme. At each time step the cell age increases. The cell will rest in quiescence if there is insufficient space to proliferate. If there is space the cell can migrate, and if there is sufficient space for the cell to divide it will proceed into mitosis. In the event of spontaneous apoptosis $\alpha$ or if the proliferation capacity $\rho$ is exhausted the cell will undergo apoptosis; otherwise it will produce a daughter cell.

\subsection{Radiotherapy model}

The fraction of cells that survive radiation is modeled using the established linear-quadratic (LQ) model:

$$
S(D)=e^{-\xi\left(\alpha D+\beta D^{2}\right)},
$$

where $S(D)$ is the surviving fraction of cells after application of dose $D=n \times d$ ( $n$ is number of fractions with dose $d$ ) and $\alpha$ and $\beta$ are tissue-specific radiosensitivity parameters. We assume $\xi=1$ for proliferating cells and $\xi<1$ for more radioresistant quiescent cells. In the discrete model we interpret the treatment survival fraction as the probability an exposed cell survives treatment [39]. For this study we will apply typical breast cancer radiosensitivity parameters $\alpha / \beta=10$ and $\alpha=0.3$ [40]. The LQ model has been extended to account for inter-fraction repopulation and reoxygenation of hypoxic cells [41]. In this study we focus on microtumors in the target area of the radiation, with the number of cancer cells being sufficiently low such that hypoxia can be ignored. Tumors smaller than diffusion limited size $(\approx 1 \mathrm{~mm}$ diameter $)$ have been found to be fully oxygenated [42]. Quiescent cells in our model arise due to intratumoral competition for space [22, 23], and become reactivated when adjacent space becomes vacant. Quiescent cell radiosensitivity is a major determinant for treatment success, and the spatial distribution of proliferating cells and their killing due to radiation plays a crucial role in the activation of quiescent cells during course of the treatment. We will use the basic LQ model to calculate the survival probability $S(d)$ for each proliferating cancer cell at each of $n$ discrete time points according to the simulated treatment protocol, and solve the tumor growth model between fractions to account for repopulation dynamics 
and re-entry of quiescent cells into cycling status. The LQ model is evaluated for quiescent cells in proportion to the quiescence radiosensitivity. In line with the cancer stem cell hypothesis, tumor cure is achieved when the cancer stem cell compartment is eradicated [15, 16] (figure 2). We therefore assume tumor cure when all cancer stem cells are eradicated, even if a cancer cell mass resides.

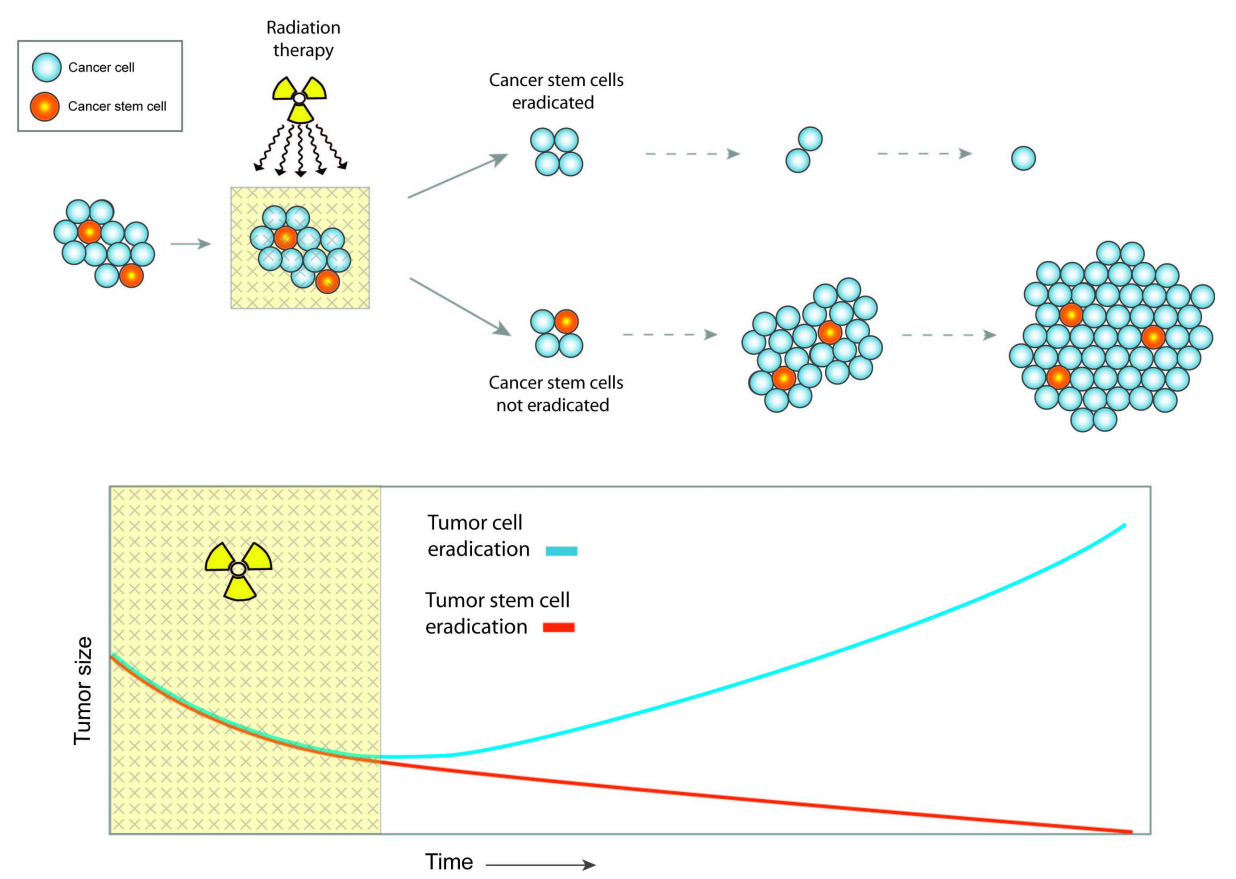

Figure 2: Cartoon of the cancer stem cell hypothesis in radiotherapy. If only a single cancer stem cell survives radiation the tumor will recur and potentially become bigger and more aggressive than it would have been without treatment. If cancer stem cells are eradicated by radiation (yellow zone), the residual tumor population will inevitably degenerate.

\section{Results}

\subsection{Effect of symmetric cancer stem cell division probability $p_{s}$ on tumor stem cell fraction and tumor growth}

We first use the model to simulate tumor development from a single cancer stem cell, and investigate how tumor growth changes as the symmetric stem cell division probability $p_{s}$ changes. For all simulations we assume a cancer cell proliferation capacity $\rho_{\max }=15$ cell divisions, cell migration $\mu=15$ cell widths per day, and a spontaneous cell death rate of $\alpha=0.01$. We solved the tumor growth model for $p_{s}=10 \%, 25 \%, 50 \%$ and $75 \%$. Due to the stochastic nature of the model we 
performed 10 independent simulations in each case and compiled the average statistics.
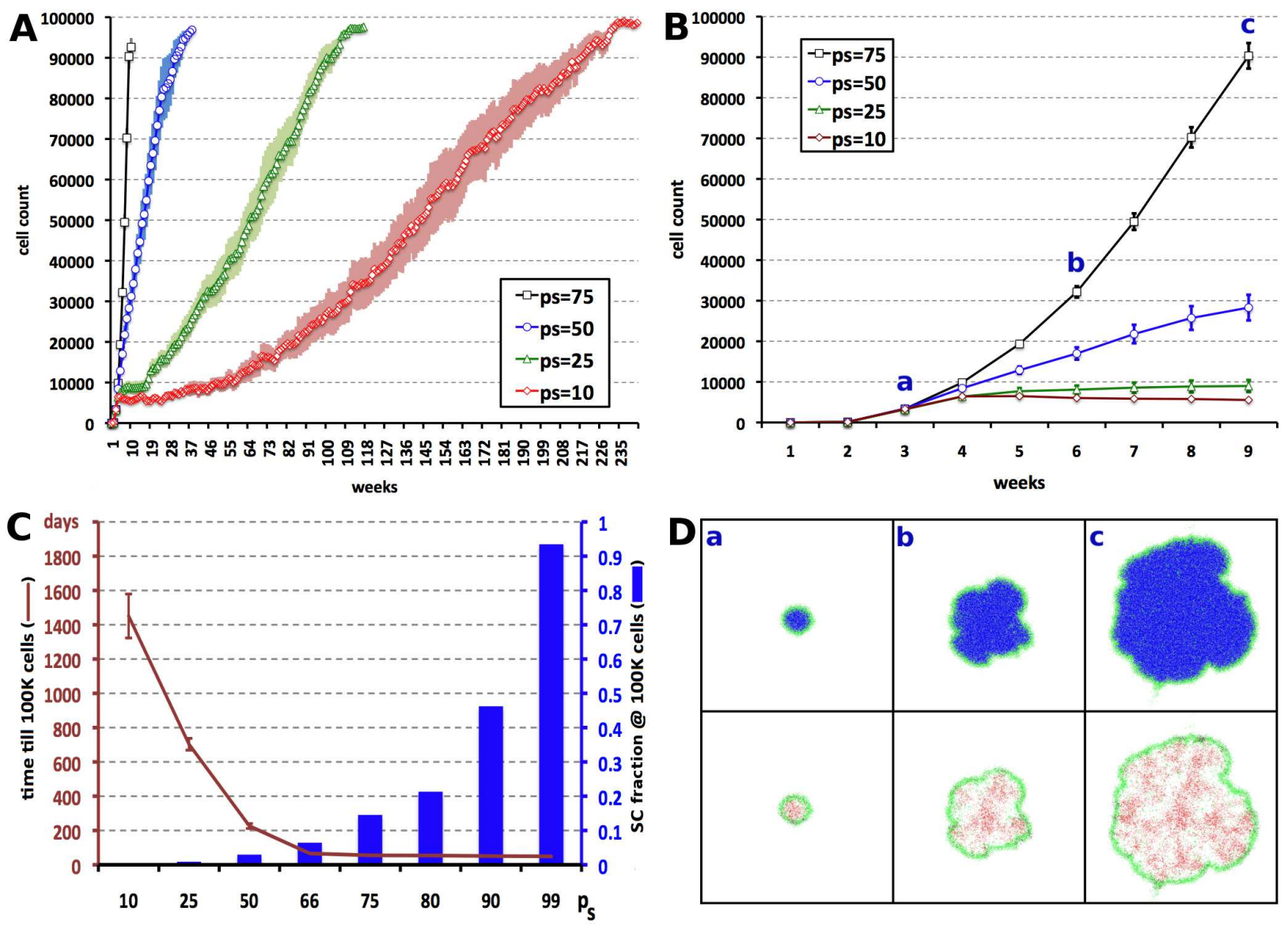

Figure 3: A. Simulations of tumor growth for different symmetric stem cell division probabilities $p_{s}=75 \%$ (black squares line), $p_{s}=50 \%$ (blue circles), $p_{s}=25 \%$ (green triangles) and $p_{s}=10 \%$ (red diamonds). Shown are averages and standard errors for 10 independent simulations each. B. First ten weeks of tumor development. C. Average times until the target size of 100, 000 cells is reached are shown (red line) along with the fractions of cancer stem cells within the tumor (blue columns) for different symmetric stem cell division probabilities $p_{s}$. D. Spatio-temporal evolution of quiescent (blue) and proliferating (green) cells and the spatial location of cancer stem cells (red) at three different time points in the aggressively growing tumor plotted in B.

In the early phase of simulated tumor growth, the cancer stem cell and its immediate progeny face no competition, and migrate to free space in accordance with the value $\mu$. Initial tumor growth is thus exponential. With increasing cell number competition for space dictates cell dynamics and cells in the core of the tumor are forced into quiescence. Only when the cells on the outer rim migrate away or die off (either because their proliferation capacity is exhausted, i.e. $\rho=0$, or due to spontaneous apoptosis $\alpha$ ) quiescent cells can become proliferative again and contribute to tumor 
growth. Crucial for the advancement of the tumor proves to be the ability of cancer stem cells to symmetrically divide to increase the number of immortal cells that can give rise to may potent daughter cells. In all simulations, despite variations in symmetric stem cell division probability $p_{s}$, tumors are able to reach the target size of 100, 000 cells. The times for the tumor to develop, however, varies, as does the final stem cell fraction. If the stem cell has a low symmetric division probability, i.e. $p_{s}=10 \%$, it takes on average 1,451 days for the tumor to reach target size. These tumors feature on average $336 \pm 19$ cancer stem cells, representing as little as $0.34 \%$ of the total tumor population. Increasing the symmetric stem cell division probability $p_{s}$ reduces the tumor development time drastically down to 55 days for $p_{s}=75 \%$. Remarkably, although the cancer stem cells divide symmetrically three out of four times, the final stem cell pool comprises only $15.19 \%$ of the total tumor $\left(14,071 \pm 515\right.$ cells). Symmetric division probabilities of $p_{s}=50 \%$ and $p_{s}=25 \%$ result in $2.65 \%$ and $1.34 \%$ cancer stem cells, respectively (Figure 3 ). Simulations of our model indicate that even if cancer stem cells preferably divide symmetrically, the fraction of cancer stem cells in the tumor is relatively small. Figure 3 (right panel) shows the spatio-temporal evolution of the distribution of proliferating and quiescent cells, as well as the spatial location of cancer stem cells in a rapidly growing tumor. In line with other findings [43], the proliferating fraction of cancer cells is almost exclusively limited to the outer rim where sufficient space is available for cells to grow and proliferate. Furthermore, the cancer stem cell compartment is not a single cluster of cancer cells but is dispersed among many small niches enriched with cancer stem cells throughout the tumor. Potent cells and cancer stem cells are almost exclusively located in the core of the tumor behind the proliferating rim and are primarily quiescent. Our simulations reveal that approximately $81 \%$ of all cells and $96 \%$ of stem cells become quiescent for $p_{s}=10 \%$. The corresponding percentages for $p_{s}=25,50$, and $75 \%$ are $82 \%(97 \%), 80 \%(95 \%)$ and $73 \%(90 \%)$, respectively. This is in line with previous observations that many of the cells in a tumor are nonproliferative but still clonogenic [44, 45]. The presence of quiescent cells is an important treatment determinant, as such cells tend to be both radio- and chemo-resistant $[26,46]$.

\subsection{Cancer stem cell pool size and radiotherapy success}

Tumors developing from stem cells with different symmetric stem cell division probabilities $p_{s}$ feature different stem cell fractions, ranging from $0.34 \%$ to $15.15 \%$ for the chosen parameter sets $\left(p_{s}=10-75 \%\right)$. Eradication of cancer stem cells is the key to successful tumor control (c.f. figure 2 ). The fewer cancer stem cells there are in a tumor, the more likely tumor control will be achieved. The majority of those stem cells, however, resides in the quiescent core of the tumor and are thus more resistant to damage. To clearly contribute radiation success or failure to stem cell pool size, we first neglect quiescent cell radioresistance [26] and assume quiescent cells are as radiosensitive as their proliferating counterparts. We also ignore intrinsic cancer stem cells radioresistance [47]. After initial analysis we restore these well-known resistance determinants and assess their specific effects on the calculated tumor control probabilities.

Figure 4 shows the radiation response of tumors and their cancer stem cells to a conventional 2 Gy $\times 25$ fractionated radiation protocol. If tumors have only a few stem cells (336 in a 100, 000- 
cell tumor; $p_{s}=10 \%$ ) the stem cell compartment is eradicated after 16 fractions on average. After 25 fractions, all tumor cells are killed. Complete tumor eradication is not achieved if initially more cancer stem cells, i.e. $\approx 1,303$ if $p_{s}=25 \%$, reside in the tumor, but the cancer stem cell compartment is completely eradicated after 24 fractions, so tumor control is achieved nonetheless. Larger numbers of initial cancer stem cells $\left(2,719\right.$ and 15,718 for $p_{s}=50 \%$ and $p_{s}=75 \%$, respectively) lead to failure of stem cell eradication and thus treatment failure.

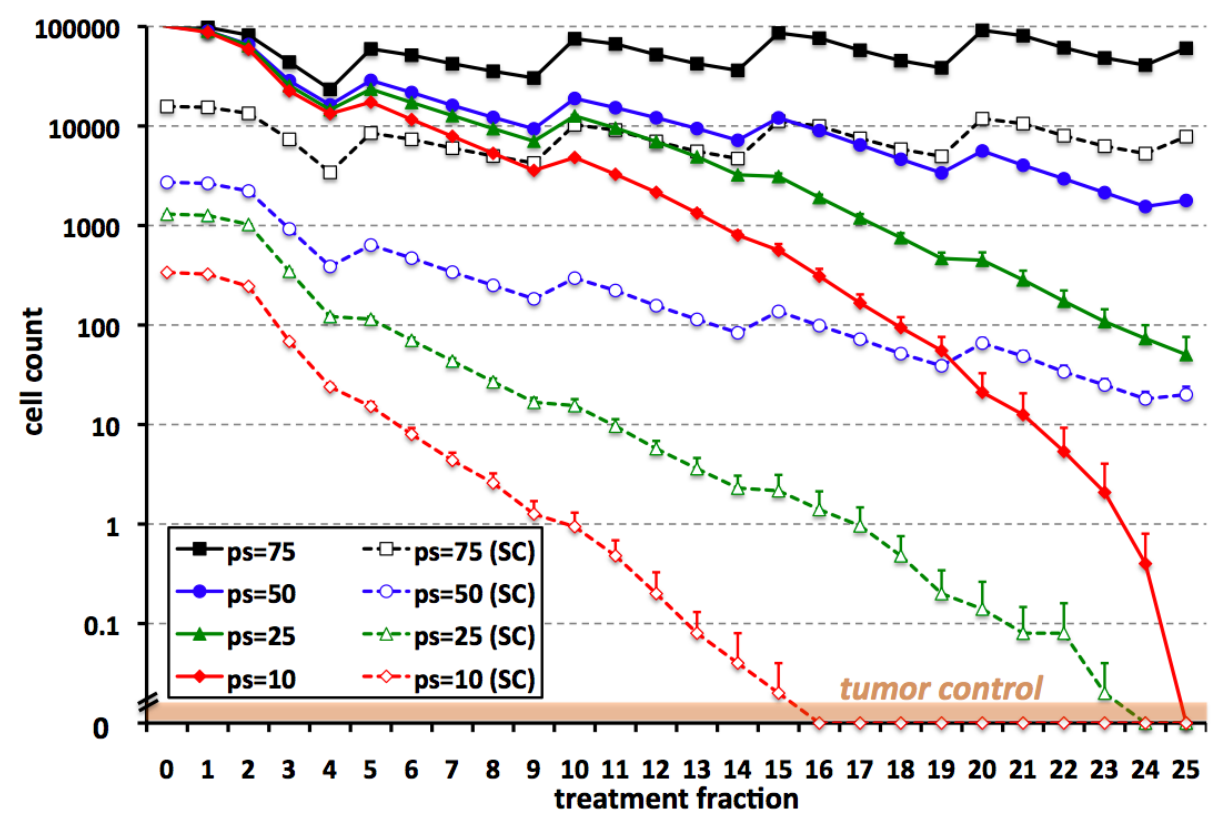

Figure 4: Population dynamics of tumors with different cancer stem cell numbers during conventional radiation treatment of 2 Gy $\times 25$ fractions. Solid plots show total number of cancer cells, dotted plots show number of cancer stem cells. $p_{s}$ values determine stem cell repopulation and correspond to initial cancer stem cell pool sizes plotted in figure 3C. Shown are averages and standard error for 50 independent simulations each.

Before considering stem cell and quiescent cell radioresistance, variations are seen in the control of tumors of identical size with homogeneous cell radiosensitivity. Successful tumor control is achieved when all cancer stem cells are depleted, which is directly related to initial number of cancer stem cells at the beginning of treatment and the production of new cancer stem cells.

\subsection{Hypofractionation and quiescence radiosensitivity}

We now use the tumor growth and radiotherapy model to evaluate the tumor cure probability of novel hypofractionated radiation protocols. The standard treatment of many solid tumors comprises the delivery of a large dose in many small increments. The time between treatment fractions minimizes late-effect damage to normal tissues, while the fractions themselves target the earlyresponding tumor tissue. In the conventional treatment with $50 \mathrm{~Gy}$, radiation is delivered in 25 
fractions of 2 Gy each (henceforth called Protocol $A$ ). Treatment is given five days per week, with no treatments on the weekends, defining the radiotherapy over a course of five weeks (i.e. 35 days). This protocol has proven successful for a number of solid tumors. However, patients need to return to the clinic to receive their radiation for many days which presents logistic problems especially for elderly patients [48]. Clinical trials with more convenient protocols are ongoing that aim to achieve similar, if not better tumor control than the standard protocol. Fewer fractions with larger doses are under consideration, with larger inter-fraction times to reduce normal tissue toxicity. The biological limitations, of course, are inter-fraction repopulation and the loss of some quadratic killing from the fractionation. These negative responses must be nullified by increased cell killing with larger doses. In this study we compare the conventional $2 \mathrm{~Gy} \times 25$ protocol with novel clinical trials of $3 \mathrm{~Gy} \times 13$ (delivered over the same time period, with three fractions per week; the UK Standardisation of Breast Radiotherapy (START) Trial A (Protocol B) [49]) and 6 Gy $\times 5$ (one fraction per week over five weeks, FAST trial (Protocol $C$ ) [50]). Due to the stochastic nature of the model we again simulate each treatment protocol ten times on five different tumors each with $p_{s}=10 \%$ and $p_{s}=25 \%$ - tumors that are controlled under Protocol $A$ (c.f. figure 4 ).

With a symmetric cancer stem cell division probability $p_{s}=10 \%$, and an initial 336 stem cells in the 100,000-cell tumors, each of 50 simulations of the conventional fractionated treatment (Protocol $A$ ) eradicates the cancer stem cell and the total tumor population (figure 5). Hypofractionation with Protocol $B$ and Protocol $C$ yield comparable residual tumor cell counts (78 and 44 cells), but the longer repopulation period in the latter protocol offsets the superior cell kill with each fraction. In one of 50 simulations of Protocol $C$, two cancer stem cells survived resulting in an average of 0.04 and an estimated tumor cure probability $\mathrm{TCP}=98 \%$, whereas all cancer stem cells are eradicated in all simulations of Protocol $B$ (figure 5). In tumors with larger stem cell compartments $\left(1,303\right.$ stem cells, $\left.p_{s}=25 \%\right)$, the number of residual tumor cells after treatment are 105, 489 and 1, 078 cells for Protocols $A, B$ and $C$, with respective cancer stem cell counts of 0, 0.06 and 0.54. The corresponding estimated tumor control probabilities (TCPs) are 100\%, 98\%, $84 \%$.

We have shown that stem cell pool size and inter-fraction repopulation influence tumor control by radiation in tumors with homogeneous radiosensitivity. Analogous to the oxygen enhancement ratio (OER) of about 3.0 [51], we introduce a radioresistance for quiescent cells by setting $\xi$ in Equation 2.1 to $\xi=0.33$ (figure 5). This results in a tumor control probability of $32 \%$ for Protocol $C$ if $p_{s}=10 \%$ (corresponding to an average 2.8 surviving cancer stem cells), whereas complete eradication of the stem cell pool is still achieved for Protocols $A$ and $B$. Tumor control values for tumors with $p_{s}=25 \%$ reduce to $90 \%, 70 \%$ and $0 \%$ for the three protocols. The tumor cure probabilities for the different treatment protocols and parameter sets are summarized in table 1.

Simulations of the presented model indicate a space - dependent impact of quiescent cell radiosensitivity. Cells that die due to radiation vacate the space they occupy, and neighboring cells compete to migrate into or place their daughter cells into the freed space. Depending on where cells die due to radiation, and how quickly previously quiescent cells can become proliferative 

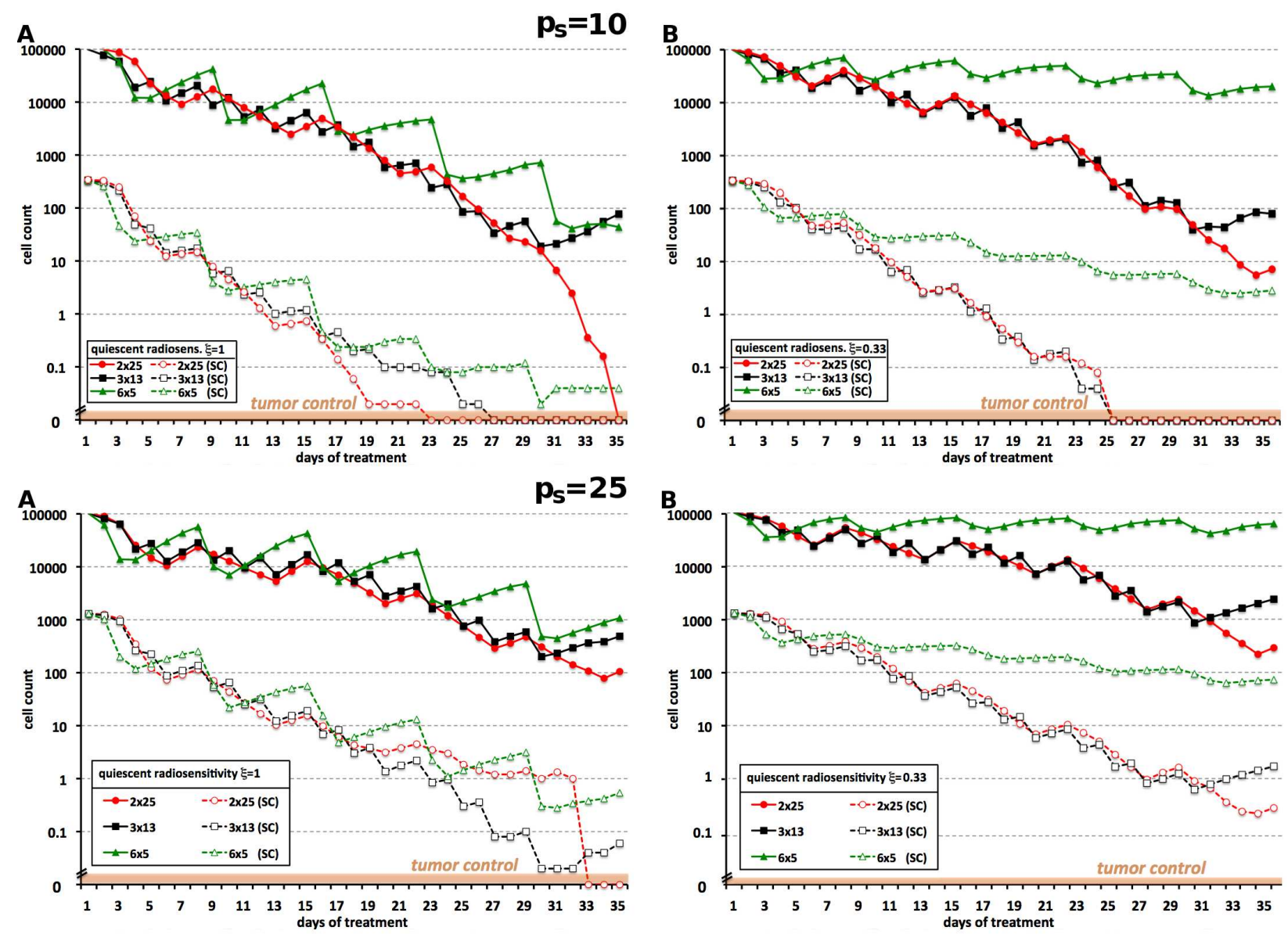

Figure 5: Tumor cell and cancer stem cell dynamics during the five weeks of radiation treatment with different protocols on tumors with symmetric stem cell division probabilities of $p_{s}=10$ (top row) and $p_{s}=25$ (bottom). Different treatment responses are shown for quiescent cell radiosensitivities $\xi=1$ ( $100 \%$ radiosensitivity; left) and $\xi=0.33$ (right).

\begin{tabular}{|c||c|c|c||c|c|c|}
\hline \multicolumn{1}{|c||}{ TCP } & \multicolumn{2}{c||}{ quiescent radiosensitivity $\xi=1$} & \multicolumn{3}{c|}{ quiescent radiosensitivity $\xi=0.33$} \\
\hline & $2 \mathrm{~Gy} \times 25$ & $3 \mathrm{~Gy} \times 13$ & $6 \mathrm{~Gy} \times 5$ & $2 \mathrm{~Gy} \times 25$ & $3 \mathrm{~Gy} \times 13$ & $6 \mathrm{~Gy} \times 5$ \\
\hline \hline $\begin{array}{c}p_{s}=10 \\
\left(s c_{f}=0.34 \%\right)\end{array}$ & 100 & 100 & 98 & 100 & 100 & 32 \\
\hline $\begin{array}{c}p_{s}=25 \\
\left(s c_{f}=1.34 \%\right)\end{array}$ & 100 & 98 & 84 & 90 & 70 & 0 \\
\hline
\end{tabular}

Table 1: Tumor Cure Probabilities (TCP) for different treatment protocols. $p_{s}$ : symmetric stem cell division rate determining stem cell fraction $s c_{f}$ within the tumor. 
again, radiation treatment either successfully eradicates the whole stem cell compartment or fails to provide tumor control. Figure 6 shows the spatial distribution of proliferating and quiescent cells after selected treatment fractions of Protocol $A$, assuming quiescent cell radiosensitivities $\xi=0$, $\xi=0.5$ and $\xi=1$. Radiation of a tumor with radioresistant, i.e. $0 \%$ radiosensitive quiescent cells yields partial eradication of the outer rim of proliferating cells only. With increasing fraction number, more space that was occupied by proliferating cells becomes vacant, and previously quiescent cells become proliferative and thus sensitive to future radiation fractions. Many fractions are necessary to eradicate a tumor with a radioresistant quiescent core. With increasing quiescent cell radiosensitivity cell kill occurs additionally in the quiescent cell compartment behind the outer rim resulting in accelerated reactivation of quiescent cells in the tumor core, which in turn yields better tumor control in fewer fractions.

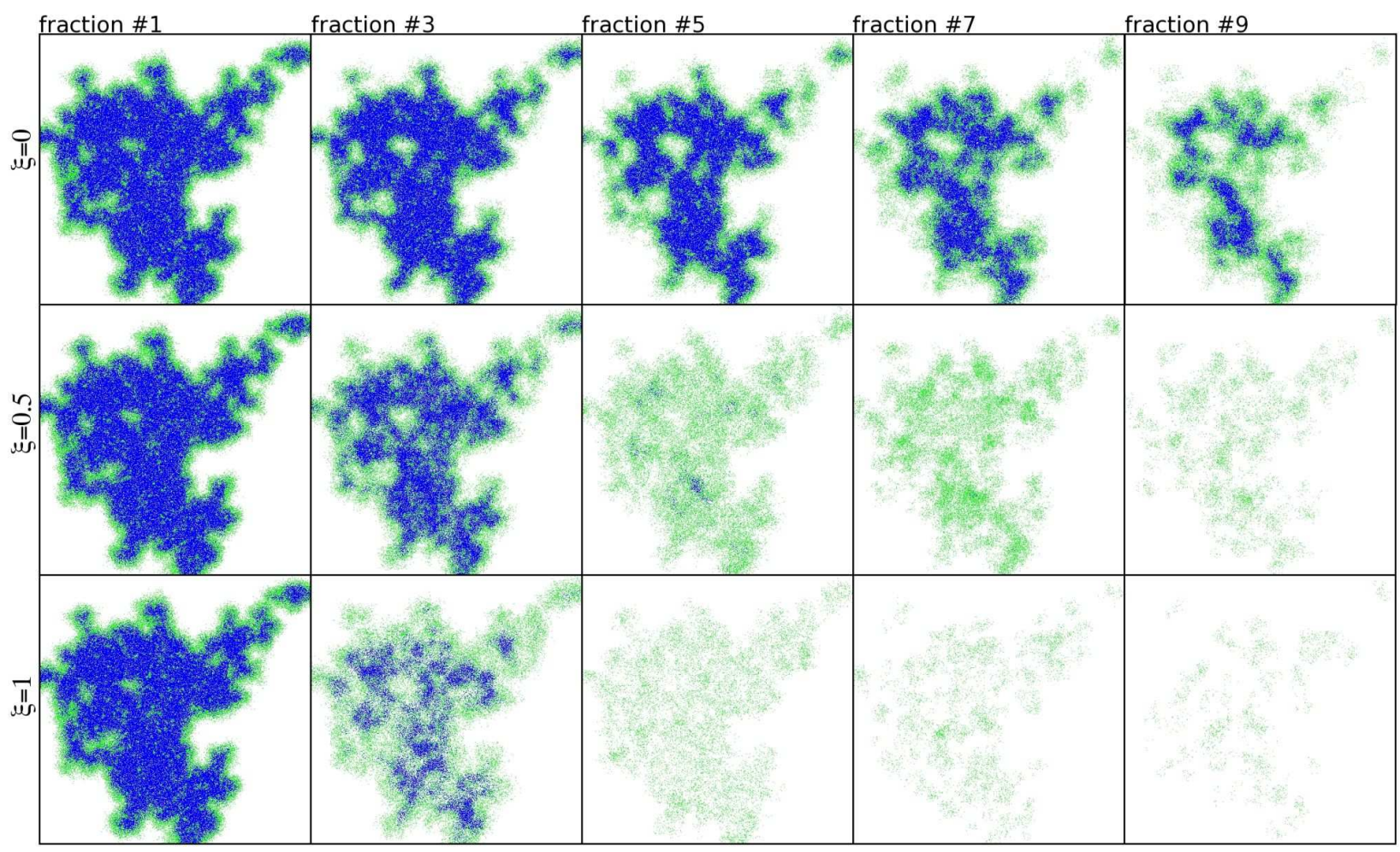

Figure 6: Representative simulation of the spatio-temporal evolution of proliferating (green) and quiescent (blue) cells during 2 Gy $\times 25$ fractionated radiotherapy with quiescent radiosensitivities $\xi=0$ (top row), $\xi=0.5$ (center row) and $\xi=1$ (bottom row). Radiation of a tumor with radioresistant quiescent cells yields partial eradication of the outer rim of proliferating cells only. With increasing quiescent cell radiosensitivity rapid reactivation of quiescent cells and thus better tumor control is achieved. 


\section{Disucssion and Conlusions}

We have presented here a single-cell-based model of tumor growth and radiotherapy. The model is motivated by the clinical question of the role in treatment outcome of cancer stem cells and quiescent tumor cells as they respond to radiation-dependent changes in their spatial distribution. All cells behave individually following intrinsic rules, constrained by available space in their local environment.

Simulations of our model revealed that tumors of identical size can develop with significant differences in the tumor-perpetuating stem cell compartment. We limit our study to symmetric stem cell division as the only mechanism to enlarge the stem cell pool, and neglect mechanisms such as cancer cell dedifferentiation [52] or epithelial-mesenchymal transition [53] to reduce complexity. We have shown that even large intrinsic symmetric cancer stem cell division probabilities result in only a small stem cell sub-population in the growing tumor. A symmetric division probability of $p_{s}=50 \%$, for example, leads to a stem cell fraction of only $2.65 \%$. The tendency of the cancer stem cell pool to be small despite a wide range of $p_{s}$ values not only supports the hypothesis that cancer stem cells are only a minority of cells in a tumor $(1 \%-0.01 \%$ [15]), but assures longer tumor development times than would have been expected if the stem cell pool size matches the symmetric division probability. This is in line with previous reports that many years can pass before a single cancer cell develops a tumor of a detectable size [54].

The model reproduces experimentally and clinically observed proliferation gradients in a tumor without introducing oxygen and nutrients as limiting factors, again in line with recent reports $[22,23]$. The core of each tumor cluster is predominantly quiescent, and only cells on the outer rim are proliferating. Such a model of tumor development with heterogeneous cell characteristics allows for realistic simulations of response to radiation treatment. Tumor cell heterogeneity is realized as cell stemness, cell proliferation capacity, and proliferative status (proliferative or quiescent), all of which bear on treatment success or failure. Both cell proliferation capacity and proliferation status change during the course of treatment. The stochastic effect of radiation on cell killing has shown significant impact on treatment success even when a certain treatment protocol is applied repeatedly to the same in silico tumor. The treatment outcome variability is due to the competition for space that arises when cells die or migrate during radiotherapy. Our model predicts that the spatial distribution of cells and the spatio-temporal evolution of the tumor cell morphology during radiation is highly stochastic and the major determinant of treatment success. The wide variability of treatment success for the same tumor indicates that more sophisticated treatment models that take better account of stochastic tumor heterogeneity would improve TCP estimation.

Simulations of radiotherapy on tumors of identical size but with varying stem cell fraction, assuming homogeneous radiosensitivity for all cancer cells, suggest that the stem cell fraction is a fundamental determinant for treatment success. Only if the number of stem cells is sufficiently small at the beginning of treatment and the inter-fractional stem cell production is low tumor control can be achieved. 
We have shown that if quiescent cells, which make up the majority of the tumor core, are more radioresistant, novel hypofractionation radiation protocols in which fewer but larger doses of radiation are delivered to the tumor are only able to achieve tumor control if 1) the stem cell population is sufficiently small and 2) symmetric division and stem cell repopulation do not antagonize the increased radiation kill in the larger, less frequent fractions. Comparison of local-regional tumor relapse in the clinic at five years after treatment revealed a 3.6\% likelihood with the conventional treatment (Protocol A, 2 Gy $\times 25$ fractions), and a $5.2 \%$ likelihood with hypofractionation (Proto$\operatorname{col} B, 3 \mathrm{~Gy} \times 13)$ [49], with comparable results expected for Protocol $C(6 \mathrm{~Gy} \times 5)$ [50]. If the tumor cure probability is directly related to local recurrence of the disease, and quiescent cells exhibit about a third of the radiosensitivity of their proliferating counterparts [51], then the stem cell fraction in a breast tumor is likely to be even less than the simulated $0.34 \%$, implying a symmetric division probability $p_{s}<10 \%$. To draw conclusions like this, however, correct tumor size, quiescent tumor fraction and radiosensitivity parameters $\alpha$ and $\beta$ in (2.1) need to be known.

In this study we have restricted tumor growth and simulation of radiotherapy to tumors of 100, 000 cells to reduce computational complexity. Tumors that can be clinically detected and treated with radiation are at least ten to twenty times bigger. Furthermore, for simplicity we have assumed that cells that are killed by radiation are removed from the system immediately, heightening the opportunity for quiescent cells to become proliferative and radiosensitive by the time of next radiation. Including a delay in radiation-induced cell death [55] could shift the tumor control accordingly, and less-frequent fractions might provide similar tumor control than the conventional treatment. In this context, an accelerated treatment with initially fewer fractions and more frequent deliveries later in the treatment might prove useful. We have explicitly ignored additional factors that are proven to be important radiobiology to draw conclusions about stem cell fraction, quiescent radiosensitivity and the importance of the evolution of the spatio-temporal proliferation-quiescent cell morphology in tumors during radiation. Further development of this work should include such higher-order mechanisms to capture the full interplay of cell kinetics controlling population response to therapy.

\section{Acknowledgements}

The authors gratefully acknowledge financial support from the American Association for Cancer Research Centennial Postdoctoral Fellowship in Cancer Research 08-40-02-ENDE (HE) and National Aeronautics and Space Administration Specialized Center of Research grant NNJ04HJ12G (HE,LH,PH), and thank Clare Lamont for her help preparing figure 2.

\section{References}

[1] D. Hanahan, R. Weinberg. The hallmarks of cancer. Cell, 100 (2000), No.1, 57-70. 
[2] P. Armitage, R. Doll. The age distribution of cancer and a multi-stage theory of carcinogenesis. Br J Cancer, 8 (1954), No.1, 1-12.

[3] W.C. Black, H.G. Welch. Advances in diagnostic imaging and overestimations of disease prevalence and the benefits of therapy. N Engl J Med, 328 (1993), No.17, 1237-1243.

[4] T. Lapidot, C. Sirard, B. Murdoch, et al. A cell initiating human acute myeloid leukaemia after transplantation into SCID mice. Nature, 367 (1994), No. 6464, 645-648.

[5] D. Bonnet, J.E. Dick. Human acute myeloid leukemia is organized as a hierarchy that originates from a primitive hematopoietic cell. Nat Med, 3 (1997), No. 7, 730-737.

[6] M. Al-Hajj, M.S. Wicha, A. Benito-Hernandez, S.J. Morrison, M.F. Clarke. Prospective identification of tumorigenic breast cancer cells. Proc Natl Acad Sci USA, 100 (2003), No.7, 3983-3988.

[7] J.E. Dick. Breast cancer stem cells revealed. Proc Natl Acad Sci USA, 100 (2003), No.7, 3547-3549.

[8] S.K. Singh, C. Hawkins, I.D. Clarke. Identification of a cancer stem cell in human brain tumors. Cancer Res, 63 (2003), No.18, 5821-5828.

[9] S.K. Singh, I.D. Clarke, M. Terasaki, et al. Identification of human brain tumour initiating cells. Nature, 432 (2004), No.7015, 396-401.

[10] D. Fioriti, M. Mischitelli, F. Di Monaco, et al. Cancer stem cells in prostate adenocarcinoma: a target for new anticancer strategies. J. Cell Physiol., 216 (2008), No.3, 571-575.

[11] N.J. Maitland, T. Colling. Prostate cancer stem cells: a new target for therapy. J. Clin. Oncol., 26 (2008), No.17, 2862-2870.

[12] M. Todaro, M. Perez Alea, A.B. Di Stefano, et al. Colon cancer stem cells dictate tumor growth and resist cell death by production of interleukin-4. Cell stem cell, 1 (2007), No.4, 389-402.

[13] P. Cammareri, Y. Lombardo, M.G. Francipane, et al. Isolation and culture of colon cancer stem cells. Methods Cell Biol., 86 (2008), 311-324.

[14] S.J. Morrison, J. Kimble. Asymmetric and symmetric stem-cell divisions in development and cancer. Nature, 41 (2006), No. 7097, 1068-1074.

[15] R. Reya, R., S.J. Morrison, M.F. Clarke, I.L. Weissman. Stem cells, cancer, and cancer stem cells. Nature, 414 (2001), No.6859, 105-111.

[16] D. Dingli, F. Michor. Successful therapy must eradicate cancer stem cells. Stem Cells, 24 (2006), No.12, 2603-2610. 
[17] R.T. Prehn. The inhibition of tumor growth by tumor mass. Cancer Res, 51 (1991), No.1, 2-4.

[18] J. Folkman. Tumor angiogenesis: therapeutic implications. N Engl J Med, 285 (1971), No.21, 1182-1186.

[19] G.N. Naumov, E. Bender, D. Zurakowski, et al. A model of human tumor dormancy: an angiogenic switch from the nonangiogenic phenotype. J Natl Cancer Inst, 98 (2006), No.5, 316-325.

[20] M.H. Barcellos-Hoff. It takes a tissue to make a tumor: epigenetics, cancer and the microenvironment. Journal of mammary gland biology and neoplasia, 6 (2001), No.2, 213-221.

[21] R. Gatenby, R.J. Gillies. A microenvironmental model of carcinogenesis. Nat Rev Cancer, 8 (2008), No.1, 56-61.

[22] A. Brú, S. Albertos, J.L. Subiza et al. The universal dynamics of tumor growth. Biophys J 85 (2003), No.5, 2948-2961.

[23] J. Galle, M. Hoffmann, G. Aust. From single cells to tissue architecture - a bottom-up approach to modelling the spatio-temporal organization of complex multi-cellular systems. $\mathbf{J}$ Math Biol 58 (2009), 261-283.

[24] L. Norton. Conceptual and practical implications of breast tissue geometry: toward a more effective, less toxic therapy. Oncologist, 10 (2005), No. 6, 370-381.

[25] L. Norton, J. Massague. Is cancer a disease of self-seeding? Nat Med, 12 (2006), No.8, $875-878$

[26] S. Masunaga, K. Ono, M. Abe. A method for the selective measurement of the radiosensitivity of quiescent cells in solid tumors - combination of immunofluorescence staining to BrdU and micronucleus assay. Radiat Res, 125 (1991), No. 3, 243-247.

[27] G.W. Barendsen, C. Van Bree, N.A.P. Franken. Importance of cell proliferative state and potentially lethal damage repair on radiation effectiveness: implications for combined tumor treatments. Int. J. Oncol., 19 (2001), No. 2, 247-256.

[28] J.J. Kim, I.F. Tannock. Repopulation of cancer cells during therapy: an important cause of treatment failure. Nat Rev Cancer, 5 (2005), No. 7, 516-25.

[29] H. Enderling, A.R.A. Anderson, M.A.J. Chaplain, A.J. Munro, J.S. Vaidya. Mathematical modelling of radiotherapy strategies for early breast cancer. J. Theor. Biol., 241 (2006), No. $1,158-171$.

[30] H. Enderling, M.A.J. Chaplain, A.R.A. Anderson, J.S. Vaidya. A mathematical model of breast cancer development, local treatment and recurrence. J. Theor. Biol., 246 (2007), No. 2, 245-259. 
[31] B. Ribba, T. Colin, S. Schnell. A multiscale mathematical model of cancer, and its use in analyzing irradiation therapies. Theor. Biol. Med. Model. 3 (2006), No.7.

[32] A. Dawson, T. Hillen. Derivation of the Tumour Control Probability (TCP) from a Cell Cycle Model. Comp. Math. Meth. Med, 7 (2006), 121-142.

[33] T.L. Jackson, H.M. Byrne. A mathematical model to study the effects of drug resistance and vasculature on the response of solid tumors to chemotherapy. Math Biosci,164 (2000), No. 1, 17-38.

[34] T. Alarcon, M.R. Owen, H.M. Byrne et al. Multiscale modelling of tumour growth and therapy: the influence of vessel normalisation on chemotherapy. Comp Math Methods Med, 7(2006), 85-119.

[35] H.M. Byrne, T. Alarcon, M.R. Owen et al. Modelling the response of vascular tumours to chemotherapy: a multiscale approach. Math Mod Meth Appl Sci, 16 (2006), No. 1, 12191241.

[36] E.S. Norris, J.R. King, H.M. Byrne. Modelling the response of spatially structured tumours to chemotherapy: drug kinetics. Math Comp Mod, 43 (2006), No. 7-8, 820-837.

[37] A.R.A. Anderson, M.A.J. Chaplain, K.A. Rejniak. Single-Cell-Based Models in Biology and Medicine. Birkhauser, Basel, 2007.

[38] P.K. Maini, D.L.S. McElwain, D.I. Leavesley. Traveling wave model to interpret a woundhealing cell migration assay for human peritoneal mesothelial cells. Tissue Eng., 10 (2004), No.(3-4), 475-482.

[39] B. Ribba, K. Marron, Z. Agur, T. Alarcon, P.K. Maini. A mathematical model of Doxorubicin treatment efficacy for non-Hodgkin's lymphoma: investigation of the current protocol through theoretical modelling results. Bull Math Biol, 67 (2005), No.1, 79-99.

[40] M. Guerrero, X. Allen Li. Analysis of a large number of clinical studies for breast cancer radiotherapy: estimation of radiobiological parameters for treatment planning. Phys Med Biol, 48 (2003), No.20, 3307-3326.

[41] D.J. Brenner, L.R. Hlatky, P.J. Hahnfeldt, E.J. Hall, R.K. Sachs. A convenient extension of the linear-quadratic model to include redistribution and reoxygenation. Int $\mathrm{J}$ Radiat Oncol Biol Phys, 32 (1995), No. 2, 379-390.

[42] J.A. Stanley, W.U. Shipley, G.G. Steele. Influence of tumour size on hypoxic fraction and therapeutic sensitivity of Lewis lung tumour. Br J Cancer, 26 (1977), No.1, 105-113.

[43] J. Folkman, M. Hochberg. Self-regulation of growth in three dimensions. J Exp Med 138 (1973) No. 4, 745-753. 
[44] J.M. Brown, A.J. Giaccia. The unique physiology of solid tumors: Opportunities (and problems) for cancer therapy. Cancer Res, 58 (1998), 1408 -1416.

[45] S. Masunaga, K. Ono, A. Takahashi, T. Ohnishi, Y. Kinashi, M. Takagaki. Radiobiological characteristics of solid tumours depending on the p53 status of the tumour cells, with emphasis on the response of intratumour quiescent cells. Eur J Cancer, 38 (2002), No. 5, 718-727.

[46] P. Ubezio, D. Cameron. Cell killing and resistance in pre-operative breast cancer chemotherapy. BMC Cancer, 8 (2008).

[47] M. Baumann, M. Krause, R. Hill. Exploring the role of cancer stem cells in radioresistance. Nat Rev Cancer, 8 (2008), No. 7, 545-554.

[48] M. Baum, J.S. Vaidya. Targeted intra-operative radiotherapy-TARGIT for early breast cancer. Ann N Y Acad Sci, 1138 (2008), 132-135.

[49] START Trialists' Group. The UK Standardisation of Breast Radiotherapy (START) Trial A of radiotherapy hypofractionation for treatment of early breast cancer: a randomised trial. Lancet Oncol, 9 (2008), No. 4, 331-341.

[50] J. Yarnold, D. Bloomeld, J. LeVay. Prospective randomized trial testing 5.7 Gy and 6.0 Gy fractions of whole breast radiotherapy in women with early breast cancer (FAST) trial. Clin Oncol, 16 (2004), S30.

[51] E.J. Hall. Radiobiology for the Radiologist 5th edn. Lippincott Williams \& Wilkins, Philadelphia, 2000.

[52] S.K. Kang, J.B. Park, S.H. Cha. Multipotent, dedifferentiated cancer stem-like cells from brain gliomas. Stem Cells Dev, 15 (2006), No. 3, 423-435.

[53] S.A. Mani, W. Guo, M.J. Liao et al. The epithelial-mesenchymal transition generates cells with properties of stem cells. Cell, 133 (2008), No. 4, 704-715.

[54] C. Guiot, P.G. Degiorgis, P.P. Delsanto et al. Does tumor growth follow a "universal law"? J Theor Biol, 225 (2003), No. 2, 147-151.

[55] A. Wichmann, B. Jaklevic, T.T. Su. Ionizing radiation induces caspase-dependent but Chk2and p53-independent cell death in Drosophila melanogaster. Proc Natl Acad Sci USA, 103 (2006), No. 26, 9952-9957. 